\title{
Analysis of Risk Factors For Sepsis And Indicators of Prognosis In Patients With Sepsis: A Retrospective Observational Study
}

Huifeng Wang

Capital Medical University

Zhiling Zhao

Capital Medical University

Zhao-hui Tong ( $\nabla$ tongzhaohuicy@sina.com )

Capital Medical University

\section{Research Article}

Keywords: Sepsis, Septic shock, Risk factors, Prognostic value

Posted Date: June 21st, 2021

DOl: https://doi.org/10.21203/rs.3.rs-577114/v1

License: (c) (i) This work is licensed under a Creative Commons Attribution 4.0 International License.

Read Full License 


\section{Abstract}

Background: To investigate the independent risk factors for sepsis and the prognostic indicators of sepsis-related mortality to guide clinical practice.

Methods: Adult patients diagnosed with sepsis in the respiratory intensive care unit (RICU), emergency ICU (EICU), and surgical ICU (SICU) of Beijing Chao-Yang Hospital, Capital Medical University, from January 2016 to April 2021 were enrolled. Comorbidities, complications, and laboratory indicators were retrospectively analyzed. Variables with a $p$ value $<0.05$ in the univariate analysis were entered into multivariate logistic regression analysis to identify the independent risk factors for sepsis. Receiver operating characteristic curve (ROC) analysis was used for those variables with $\mathrm{P}<0.05$ in multivariate regression to evaluate the fit of the predictive model and its prognostic efficacy.

Results: A total of 123 adult patients with sepsis were enrolled, with 80 males and 43 females and a mean age of $61.56 \pm 17.12$ years. Acute respiratory distress syndrome (ARDS) occurred in 84 patients $(68.3 \%)$, acute kidney injury (AKI) occurred in 28 patients (22.8\%), acute myocardial injury (AMI) occurred in 6 patients (4.9\%), disseminated intravascular coagulation (DIC) occurred in 14 patients $(11.4 \%)$, septic shock occurred in 40 patients (32.5\%), and 41 patients (33.3\%) died. Multivariate logistic regression analysis showed that mean arterial pressure (MAP), acute physiology and chronic health evaluation II (APACHE II) score, albumin level, and the presence of DIC were independent risk factors for sepsis $(\mathrm{P}<$ 0.05). The area under the ROC curve for the model including MAP, albumin, and APACHE II score was the highest at 0.890 .

Conclusion: The MAP, APACHE II score, albumin level, and DIC were independent risk factors for sepsis. The inclusion of the MAP, albumin level, and APACHE II score in the model yielded the most accurate prediction of the risk of mortality.

\section{Introduction}

Sepsis is a common and often fatal syndrome characterized by physiological, biological, and biochemical abnormalities caused by dysregulated inflammatory responses to infection. [1, 2] Sepsis remains the leading cause of death in patients admitted to the intensive care unit (ICU).[3] The related mortality rate remains high worldwide,[4] especially in cases of septic shock.[5] Carolin et al performed a meta-analysis of 27 studies in high-income countries and found that the incidence of sepsis was 288 cases per 100,000 person-years. A multicenter cohort study in Korea reported that the incidence of sepsis in the emergency department was $1.5 \%$, and $36.5 \%$ of those patients developed septic shock.[6, 7] Several studies have shown that sepsis-related mortality has decreased every year due to the launch of the Surviving Sepsis Campaign (SSC), but there is still a high mortality rate of up to $80 \%$ in low-middleincome countries.[8] Thus, the diagnosis and treatment of sepsis need more attention.

According to numerous studies, the perception of sepsis has gradually changed from an inflammatory response syndrome to organ dysfunction. Previous studies have focused on the value of 258 different 
biomarkers, such as interleukin-27 (IL-27), interleukin-6 (IL-6), and inter-alpha inhibitor proteins, for the diagnosis, treatment and prognostic prediction of sepsis. These biomarkers have some value, but most of them are expensive and cannot be widely used in clinical practice. In addition, the role of most biomarkers in the management of patients with sepsis has not been clarified, and thus far, the most accurate combination of the commonly used clinical indicators has yet to be determined.

Considering the high incidence and mortality rate of sepsis, coupled with the limitations of the existing biomarkers, it is necessary to identify the early independent risk factors for sepsis and explore the specific prognostic indicators, which can be used to evaluate the therapeutic effect. Therefore, this study identified the independent risk factors for sepsis and the prognostic indicators to determine biomarkers that can provide valuable, clinically relevant information and guide clinical practice.

\section{Methods}

\section{Study design and participants}

We performed a retrospective cohort analysis in which patients who met the inclusion criteria and were admitted to ICU were enrolled. Adult patients diagnosed with sepsis in the respiratory intensive care unit (RICU), emergency intensive care unit (EICU) and surgical intensive care unit (SICU) of Beijing Chao-Yang Hospital, Capital Medical University from January 2016 to April 2021 were enrolled.

The inclusion criteria were as follows: (1) subjects aged above 18 years; (2) subjects who met the Sepsis 3.0 diagnostic criteria.[5]

The exclusion criteria were as follows:(1) subjects who died within 24 hours after admission; (2) subjects whose clinical data were missing or who did not undergo the relevant examinations during hospitalization; (3) subjects who were pregnant or lactating; and (4) subjects who were discharged automatically without a record of the clinical outcome.

This study was approved by the Institutional Review Board of Beijing Chaoyang Hospital (project approval number 2016-Ke-115). Informed consent from each patient was waived since we collected and analyzed all of the data retrospectively.

\section{Data collection}

Parents were divided into the survival group and the death group according to the 28-day outcome. The demographic and clinical data of the patients were entered into an electronic case report form included the following variables: basic characteristics, such as age, sex, comorbidities (e.g., hypertension, diabetes, etc.), infection site (respiratory system, urinary system, digestive system, skin and soft tissue, blood, catheter); relevant clinical indicators, such as the oxygenation index $\left(\mathrm{PaO}_{2} / \mathrm{FiO}_{2}\right)$, mean arterial pressure (MAP), arterial blood lactate level, white blood cell count (WBC), platelet count, neutrophil to lymphocyte ratio, D-dimer level, fibrinogen level, activated partial thromboplastin time (APTT), prothrombin time (PT), thrombin time (TT), international normalized ratio (INR), serum albumin level, total bilirubin level, serum 
creatinine level, blood sodium level, blood potassium level, procalcitonin level, C-reactive protein (CRP) level, Glasgow Coma Scale (GCS) score, sequential organ failure assessment (SOFA) score, acute physiology and chronic health evaluation (APACHE II) score; and outcomes, namely, septic shock, acute respiratory distress syndrome (ARDS), acute kidney injury (AKI), acute myocardial injury (AMI), acute liver injury, and disseminated intravascular coagulation (DIC).

\section{Assessment measures}

Sepsis (Sepsis 3.0): Patients with sepsis were defined as patients with infections or strongly suspected infections in the ICU with an increase in their SOFA score $\geq 2$ points from baseline.

Septic shock: Septic shock is a clinically defined subset of sepsis cases wherein, despite adequate fluid resuscitation, patients have hypotension requiring vasopressors to maintain a MAP above $65 \mathrm{mmHg}$ and have an elevated serum lactate concentration greater than $2 \mathrm{mmol} / \mathrm{L}$.

SOFA: The SOFA score separately evaluates the condition of the following organ systems: respiration, coagulation, liver, cardiovascular, central nervous, and renal.[9]

APACHE II: The APACHE II score is the sum of three items: the Acute Physiology Score (APS) (0-60 points), the age score ( $0-6$ points), and Chronic Health Evaluation (CHS) score (0-5 points). [10]

\section{Statistical analysis}

In this study, SPSS 26.0 software was used for the statistical analysis. Categorical variables were summarized using frequencies and percentages, and continuous data are presented as the means \pm SDs for normally distributed variables and the medians (interquartile ranges [IQRs]) for nonnormally distributed variables. The Mann-Whitney $U$ test was used for continuous variables, and the $\chi 2$ test or Fisher's exact test was used for categorical variables. Variables with a $p$ value $<0.05$ in the univariate analysis were entered into multivariate logistic regression analysis to identify the independent risk factors for sepsis. Receiver operating curve (ROC) analysis was used for those variables with $P<0.05$ in multivariate regression to evaluate the fit of the prediction model and the prognostic efficacy of the independent risk factors. The prognostic value was evaluated according to the area under the curve (AUC), and those variables with AUC $>0.5$ were jointly evaluated. All statistical tests were two-sided.

\section{Results}

A total of 135 consecutive patients were included from January 2016 to April 2021, 12 subjects did not meet the inclusion criteria. Thus, 123 patients were eligible for the analysis. Among them, there were 80 males and 43 females, the patients were had a mean age of $61.56 \pm 17.12$ years.

\section{Basic characteristic of the patients with sepsis}

The basic characteristics are summarized in Table 1. There were no significant differences in comorbidities, sex, neutrophil to lymphocyte ratio, D-D, procalcitonin level, CRP level, creatinine level, and 
total bilirubin level between the two groups $(P>0.05)$. The APACHEIl score, age, MAP, oxygenation index, platelet count, WBC count, arterial blood lactate level, SOFA score, albumin level, APTT, PT, TT, and INR were significantly different between the two groups $(P<0.05)$. In terms of complications, there were significant differences in the incidences of ARDS, AKI, ACl, and DIC between the two groups $(P<0.05)$. The infection sites were significantly different with regard to infections originating from the respiratory and urinary systems between the two groups $(P<0.05)$. 
Table 1

Baseline characteristics of sepsis patients

\begin{tabular}{|c|c|c|c|c|}
\hline & Total $(n=123)$ & Survival $(n=82)$ & Death $(n=41)$ & $\begin{array}{l}\text { P- } \\
\text { value }^{a}\end{array}$ \\
\hline Male, n (\%) & $80(65.0)$ & $51(62.2)$ & $29(70.7)$ & 0.349 \\
\hline Age, year (SD) & $61.56(17.12)$ & $59.47(18.33)$ & 65.68 (13.73) & 0.038 \\
\hline \multicolumn{5}{|l|}{ Laboratory index } \\
\hline $\mathrm{PaO} 2 / \mathrm{FiO} 2$ & $218.6(144.4,316.4)$ & $\begin{array}{l}243.3(168.5- \\
371.9)\end{array}$ & $\begin{array}{l}157.8(98.3- \\
220.0)\end{array}$ & $<0.001$ \\
\hline Lac, mmol/L & $1.5(1.1-2.5)$ & $1.3(1.0-1.9)$ & $2.2(1.5-3.4)$ & $<0.001$ \\
\hline MAP, mmHg & $77.2(67.8-89.3)$ & $82.7(73.0-91.8)$ & $68.0(64.3-76.7)$ & $<0.001$ \\
\hline NLR & $14.1(7.7-23.5)$ & $14.8(9.0-23.7)$ & $12.7(7.1-22.7)$ & 0.893 \\
\hline PLT, 1000/mm3 & $124(79-178)$ & $\begin{array}{l}140.0(96.5- \\
186.5)\end{array}$ & $99.0(58.0-155.0)$ & 0.01 \\
\hline WBC, $1000 / \mathrm{mm} 3$ & $12.4(7.4-17.5)$ & $11.6(7.0-15.2)$ & $16.2(10.6-20.0)$ & 0.03 \\
\hline$D-D, n g / L$ & $3.5(1.6-6.6)$ & $3.4(1.4-6.0)$ & $4.2(2.6-7.1)$ & 0.074 \\
\hline $\mathrm{Fbg}, \mathrm{mg} / \mathrm{dl}(\mathrm{SD})$ & 409 (183) & $449.1(156.6)$ & $327.2(207.7)$ & 0.014 \\
\hline APTT, s & $35.3(30.1-41.7)$ & $\begin{array}{l}33.7(28.8- \\
38.7)\end{array}$ & $41.3(32.6-48.8)$ & 0.001 \\
\hline PT, s & $13.6(12.4-15.2)$ & $\begin{array}{l}13.0(12.1- \\
14.5)\end{array}$ & $14.5(13.2-18.1)$ & $<0.001$ \\
\hline TT, s & $19.2(17.8-21.5)$ & $\begin{array}{l}18.9(17.7- \\
20.2)\end{array}$ & $21.3(18.3-23.5)$ & 0.005 \\
\hline INR & $1.2(1.0-1.3)$ & $1.1(1.0-1.2)$ & $1.2(1.1-1.5)$ & $<0.001$ \\
\hline $\mathrm{Cr}, \mathrm{umol} / \mathrm{L}$ & $100.3(68.8-167.3)$ & $\begin{array}{l}95.9(65.3- \\
160.9)\end{array}$ & $\begin{array}{l}107.2(76.8- \\
190.4)\end{array}$ & 0.120 \\
\hline
\end{tabular}

Data are presented as median (interquartile range) or $\mathrm{n}(\%)$.

Abbreviations: Lac, lactate; MAP, mean arterial pressure; NLR, neutral lymphocyte ratio; PLT, Platelet; WBC, white blood cell count; D-D, D-dimer; Fbg, fibrinogen; APTT, activated prothrombin time; PT, prothrombin time; TT, thrombin time; INR, international normalized ratio; $\mathrm{Cr}$, serum creatinine; Tbil, total bilirubin; ALB, albumin; APACHEII, acute physiology and chronic health evaluation; PCT, procalcitonin; CRP, C-reaction protein; CKD, chronic kidney disease; CHF, chronic heart failure; CAD, coronary artery disease; COPD, chronic obstructive pulmonary disease; ARDS, Acute respiratory distress syndrome; AKI, acute kidney injury; ALI, acute liver injury; ACl, acute cardiac injury; DIC, disseminated intravascular coagulation.

a Comparison between survival and death groups. 


\begin{tabular}{|c|c|c|c|c|}
\hline & Total $(n=123)$ & Survival $(n=82)$ & Death $(n=41)$ & $\begin{array}{l}\text { P- } \\
\text { value }^{a}\end{array}$ \\
\hline Tbil, umol/L & $14.5(8.7-28.7)$ & $14.3(9.0-24.1)$ & $15.7(8.3-39.3)$ & 0.211 \\
\hline ALB, $g / L$ & $28.7(26.4-32.5)$ & $29.4(27.0-33.1)$ & $27.8(25.5-30.4)$ & 0.011 \\
\hline BNP, pg/mL & $381.0(113-1005)$ & $\begin{array}{l}274.0(99.0- \\
664.0)\end{array}$ & $\begin{array}{l}717.5(275.0- \\
2399.0)\end{array}$ & 0.005 \\
\hline SOFA & $6(4-10)$ & $5(3-7)$ & $10(8-13)$ & $<0.001$ \\
\hline APACHE II & $15(10-21)$ & $13(9-16.5)$ & $23(18-26)$ & $<0.001$ \\
\hline PCT, ng/ml & $13.2(2.6-29.8)$ & $14.6(2.9-44.6)$ & $8.8(2.2-25.4)$ & 0.104 \\
\hline CRP, mg/dl & $13.2(10.1-17.0)$ & $13.4(11.0-17.4)$ & $13.1(8.7-16.8)$ & 0.278 \\
\hline \multicolumn{5}{|l|}{ Comorbidities } \\
\hline Hypertension, n (\%) & $62(50.4)$ & $41(50.0)$ & $21(51.2)$ & 0.899 \\
\hline Diabetes, n (\%) & $42(34.1)$ & $27(32.9)$ & $15(36.6)$ & 0.687 \\
\hline CKD, n (\%) & $24(19.5)$ & 15 (18.3) & $9(22.0)$ & 0.629 \\
\hline CHF, n (\%) & 19 (15.4) & $13(15.9)$ & $6(14.6)$ & 0.86 \\
\hline CAD, n (\%) & $25(20.3)$ & $14(17.0)$ & $11(26.8)$ & 0.205 \\
\hline Arrhythmia, n (\%) & 19 (15.4) & $12(14.6)$ & 7 (17.1) & 0.724 \\
\hline $\begin{array}{l}\text { Cerebrovascular Disease, } \mathrm{n} \\
(\%)\end{array}$ & $19(15.4)$ & $12(14.6)$ & $7(17.1)$ & 0.724 \\
\hline COPD, n (\%) & $10(8.1)$ & $5(6.1)$ & $5(12.2)$ & 0.299 \\
\hline $\begin{array}{l}\text { Organ transplantation, } \mathrm{n} \\
(\%)\end{array}$ & $8(6.5)$ & $4(4.9)$ & $4(9.8)$ & 0.439 \\
\hline Hemopathy , n (\%) & $4(3.3)$ & $1(1.2)$ & $3(7.3)$ & 0.107 \\
\hline Malignance, n (\%) & $7(5.7)$ & $4(4.9)$ & $3(7.3)$ & 0.685 \\
\hline
\end{tabular}

Data are presented as median (interquartile range) or $\mathrm{n}(\%)$.

Abbreviations: Lac, lactate; MAP, mean arterial pressure; NLR, neutral lymphocyte ratio; PLT, Platelet; WBC, white blood cell count; D-D, D-dimer; Fbg, fibrinogen; APTT, activated prothrombin time; PT, prothrombin time; TT, thrombin time; INR, international normalized ratio; $\mathrm{Cr}$, serum creatinine; Tbil, total bilirubin; ALB, albumin; APACHEII, acute physiology and chronic health evaluation; PCT, procalcitonin; CRP, C-reaction protein; CKD, chronic kidney disease; CHF, chronic heart failure; CAD, coronary artery disease; COPD, chronic obstructive pulmonary disease; ARDS, Acute respiratory distress syndrome; AKI, acute kidney injury; ALI, acute liver injury; ACl, acute cardiac injury; DIC, disseminated intravascular coagulation.

${ }^{\text {a }}$ Comparison between survival and death groups. 


\begin{tabular}{|c|c|c|c|c|}
\hline & Total $(n=123)$ & Survival $(n=82)$ & Death $(n=41)$ & $\begin{array}{l}\text { P- } \\
\text { value }^{a}\end{array}$ \\
\hline \multicolumn{5}{|l|}{ Source of infection } \\
\hline Lung, n (\%) & $74(60.2)$ & $43(52.4)$ & $31(75.6)$ & 0.016 \\
\hline Urinary tract, n (\%) & $23(18.7)$ & $19(23.2)$ & $4(9.8)$ & 0.088 \\
\hline Abdomen, n (\%) & $26(21.1)$ & $19(23.2)$ & $7(17.1)$ & 0.416 \\
\hline Skin, n (\%) & $6(4.9)$ & $4(4.9)$ & $2(4.9)$ & 1.000 \\
\hline Blood, n (\%) & $21(17.1)$ & $12(14.6)$ & $9(22.0)$ & 0.309 \\
\hline Catheter, n (\%) & $3(2.4)$ & $2(2.4)$ & $1(2.4)$ & 1.000 \\
\hline \multicolumn{5}{|l|}{ Complications } \\
\hline ARDS, n (\%) & $84(68.3)$ & $49(59.8)$ & $35(85.4)$ & 0.004 \\
\hline $\mathrm{AKI}, \mathrm{n}(\%)$ & $28(22.8)$ & $8(9.8)$ & $20(48.8)$ & $<0.001$ \\
\hline ALI, n (\%) & $10(8.1)$ & $4(4.9)$ & $6(14.6)$ & 0.082 \\
\hline $\mathrm{ACl}, \mathrm{n}(\%)$ & $6(4.9)$ & $1(1.2)$ & $5(12.2)$ & 0.016 \\
\hline DIC, n (\%) & $14(11.4)$ & $3(3.7)$ & $11(26.8)$ & $<0.001$ \\
\hline \multicolumn{5}{|c|}{ Data are presented as median (interquartile range) or $n(\%)$. } \\
\hline \multicolumn{5}{|c|}{$\begin{array}{l}\text { Abbreviations: Lac, lactate; MAP, mean arterial pressure; NLR, neutral lymphocyte ratio; PLT, Platelet; } \\
\text { WBC, white blood cell count; D-D, D-dimer; Fbg, fibrinogen; APTT, activated prothrombin time; PT, } \\
\text { prothrombin time; TT, thrombin time; INR, international normalized ratio; Cr, serum creatinine; Tbil, } \\
\text { total bilirubin; ALB, albumin; APACHEIl, acute physiology and chronic health evaluation; PCT, } \\
\text { procalcitonin; CRP, C-reaction protein; CKD, chronic kidney disease; CHF, chronic heart failure; CAD, } \\
\text { coronary artery disease; COPD, chronic obstructive pulmonary disease; ARDS, Acute respiratory } \\
\text { distress syndrome; AKI, acute kidney injury; ALI, acute liver injury; ACl, acute cardiac injury; DIC, } \\
\text { disseminated intravascular coagulation. }\end{array}$} \\
\hline \multicolumn{5}{|c|}{${ }^{\text {a }}$ Comparison between survival and death groups. } \\
\hline
\end{tabular}

\section{Univariate and multivariate analysis of risk factors for mortality in patients with sepsis}

Variables with significant differences between the two groups were included in univariate logistic regression analyses, and the results showed that the oxygenation index, arterial blood lactate level, MAP, WBC, fibrinogen level, APTT, PT, INR, albumin level, SOFA score, APACHE II score, respiratory system infection, and incidences of ARDS, AKI, ACl, and DIC were significantly different within the two groups ( $P$ $<0.05)$.

The significant variables in the univariate regression analysis were included in the multivariate logistic regression analysis to yield an adjusted OR. The results showed that the MAP $(\mathrm{OR}, 0.889,95 \% \mathrm{Cl}, 0.822-$ $0.961, \mathrm{P}<0.05)$, APACHE II score $(\mathrm{OR}, 1.356,95 \% \mathrm{Cl}, 1.119-1.643, \mathrm{P}<0.05)$, albumin level $(\mathrm{OR}, 0.755,95 \%$ 
$\mathrm{Cl}, 0.583-0.979, \mathrm{P}<0.05)$, and $\mathrm{DIC}(\mathrm{OR}, 0.055,95 \% \mathrm{Cl}, 0.005-0.638, \mathrm{P}<0.05)$ were independent risk factors for sepsis $(P<0.05)$ (Table 2$)$. 
Table 2

Univariate analysis and multivariate analysis

\begin{tabular}{|c|c|c|c|c|c|c|}
\hline \multirow[t]{2}{*}{ Variables } & \multicolumn{3}{|c|}{ Univariate analysis } & \multicolumn{3}{|c|}{ Multivariate analysis } \\
\hline & OR & $95 \% \mathrm{Cl}$ & P-value & OR & $95 \% \mathrm{Cl}$ & P-value \\
\hline Age, years & 1.023 & $0.999-1.047$ & 0.061 & - & - & - \\
\hline \multicolumn{7}{|c|}{ Laboratory index } \\
\hline $\mathrm{PaO}_{2} / \mathrm{FiO}_{2}$ & 0.990 & $0.985-0.995$ & $<0.001$ & 0.989 & $0.978-1.000$ & 0.054 \\
\hline Lac, mmol/L & 1.482 & $1.105-1.988$ & $<0.001$ & 0.955 & $0.647-1.407$ & 0.814 \\
\hline MAP, mmHg & 0.929 & $0.896-0.963$ & $<0.001$ & 0.889 & $0.822-0.961$ & 0.003 \\
\hline PLT & 0.994 & $0.989-1.000$ & 0.054 & - & - & - \\
\hline WBC & 1.069 & $1.017-1.124$ & 0.008 & 1.123 & $0.994-1.268$ & 0.062 \\
\hline $\mathrm{D}-\mathrm{D}, \mathrm{ng} / \mathrm{ml}$ & 1.000 & $1.000-1.000$ & 0.148 & - & - & - \\
\hline $\mathrm{Fbg}, \mathrm{mg} / \mathrm{dl}$ & 0.997 & $0.995-0.999$ & 0.007 & 1.002 & $0.999-1.006$ & 0.238 \\
\hline APTT, s & 1.039 & $1.010-1.069$ & 0.008 & 0.920 & $0.855-0.991$ & 0.068 \\
\hline $\mathrm{PT}, \mathrm{s}$ & 1.273 & $1.084-1.495$ & 0.003 & 2.256 & $0.751-6.777$ & 0.147 \\
\hline $\mathrm{TT}, \mathrm{s}$ & 1.032 & $0.991-1.075$ & 0.129 & - & - & - \\
\hline INR & 11.560 & $2.069-64.582$ & 0.005 & 0.001 & $0.000-23.344$ & 0.171 \\
\hline ALB, $g / L$ & 0.890 & $0.814-0.973$ & 0.011 & 0.755 & $0.583-0.979$ & 0.034 \\
\hline BNP, pg/mL & 1.000 & $1.000-1.000$ & 0.193 & - & - & - \\
\hline SOFA & 1.516 & $1.305-1.760$ & $<0.001$ & 0.787 & $0.557-1.113$ & 0.175 \\
\hline APACHEII & 1.225 & $1.132-1.325$ & $<0.001$ & 1.356 & $1.119-1.643$ & 0.002 \\
\hline \multicolumn{7}{|l|}{ Infection site } \\
\hline Lung & 0.365 & $0.158-0.842$ & 0.018 & 0.487 & $0.092-2.572$ & 0.397 \\
\hline Urinary tract & 2.790 & $0.881-8.829$ & 0.081 & - & - & - \\
\hline
\end{tabular}

Complications

Variables with $p$-values less than 0.05 were selected to enter univariate regression analysis, and those with $p$-values less than 0.05 were included in the multivariate regression analysis.

Abbreviations: Lac, lactate; MAP, mean arterial pressure; PLT, Platelet; WBC, white blood cell count; DD, D-dimer; Fbg, fibrinogen; APTT, activated prothrombin time; PT, prothrombin time; TT, thrombin time; INR, international normalized ratio; ALB, albumin; SOFA, Sequential Organ Failure Assessment; APACHEII, acute physiology and chronic health evaluation; ARDS, Acute respiratory distress syndrome; AKI, acute kidney injury; $\mathrm{ALI}$, acute liver injury; $\mathrm{ACl}$, acute cardiac injury; DIC, disseminated intravascular coagulation; "-" not available. 


\begin{tabular}{|c|c|c|c|c|c|c|}
\hline \multirow[t]{2}{*}{ Variables } & \multicolumn{3}{|c|}{ Univariate analysis } & \multicolumn{3}{|c|}{ Multivariate analysis } \\
\hline & OR & $95 \% \mathrm{Cl}$ & P-value & OR & $95 \% \mathrm{Cl}$ & P-value \\
\hline ARDS & 0.255 & $0.096-0.673$ & 0.006 & 1.309 & $0.123-13.940$ & 0.823 \\
\hline AKI & 0.114 & $0.044-0.294$ & $<0.001$ & 0.182 & $0.023-1.452$ & 0.108 \\
\hline ALI & 0.299 & $0.079-1.127$ & 0.075 & - & - & - \\
\hline $\mathrm{ACl}$ & 0.089 & $0.010-0.788$ & 0.030 & 0.046 & $0.001-2.464$ & 0.130 \\
\hline DIC & 0.104 & $0.027-0.397$ & 0.001 & 0.055 & $0.005-0.638$ & 0.020 \\
\hline \multicolumn{7}{|c|}{$\begin{array}{l}\text { Variables with p-values less than } 0.05 \text { were selected to enter univariate regression analysis, and those } \\
\text { with p-values less than } 0.05 \text { were included in the multivariate regression analysis. }\end{array}$} \\
\hline \multicolumn{7}{|c|}{$\begin{array}{l}\text { Abbreviations: Lac, lactate; MAP, mean arterial pressure; PLT, Platelet; WBC, white blood cell count; D- } \\
\text { D, D-dimer; Fbg, fibrinogen; APTT, activated prothrombin time; PT, prothrombin time; TT, thrombin time; } \\
\text { INR, international normalized ratio; ALB, albumin; SOFA, Sequential Organ Failure Assessment; } \\
\text { APACHEII, acute physiology and chronic health evaluation; ARDS, Acute respiratory distress } \\
\text { syndrome; AKI, acute kidney injury; ALI, acute liver injury; ACl, acute cardiac injury; DIC, disseminated } \\
\text { intravascular coagulation; "-" not available. }\end{array}$} \\
\hline
\end{tabular}

\section{Prognostic value of clinical parameters in patients with sepsis}

In terms of the prognostic value of the individual indicators, the area under the ROC curve for the predication of mortality in patients with sepsis based on the MAP was $0.747(95 \% \mathrm{Cl}, 0.654-0.839, \mathrm{P}<$ $0.001)$, that based on the APACHE II score was $0.839(95 \% \mathrm{Cl}, 0.760-0.917, \mathrm{P}<0.001)$, and that based on the albumin level was $0.641(95 \% \mathrm{Cl}, 0.541-0.741, \mathrm{P}=0.011)$. (Table 3).

Table 3

AUC of the predictive variables

\begin{tabular}{|llll|}
\hline Variables & AUC & $95 \% \mathrm{Cl}$ & P-Value \\
\hline MAP & 0.747 & $0.654-0.839$ & $<0.001$ \\
\hline APACHE II & 0.839 & $0.760-0.917$ & $<0.001$ \\
\hline ALB & 0.641 & $0.541-0.741$ & 0.011 \\
\hline MAP + APACHE II & 0.878 & $0.815-0.941$ & $<0.001$ \\
\hline MAP + ALB & 0.774 & $0.690-0.858$ & $<0.001$ \\
\hline ALB + APACHE II & 0.848 & $0.777-0.919$ & $<0.001$ \\
\hline MAP + ALB + APACHE II & 0.890 & $0.832-0.948$ & $<0.001$ \\
\hline $\begin{array}{l}\text { Abbreviations: MAP, mean arterial pressure; APACHE II, acute physiology and chronic health } \\
\text { evaluation; ALB, albumin. }\end{array}$ & & \\
\hline
\end{tabular}


The above indicators with an AUC greater than 0.6 were combined to improve the predictive value. The predictive model incorporating the MAP, APACHE II score and albumin level had the highest predictive power, with an AUC of $0.890(95 \% \mathrm{Cl}, 0.832-0.948, \mathrm{P}<0.001)$. (Fig. 1)

The optimal cut-off value of MAP to evaluate prognose was $73 \mathrm{mmHg}$. The sensitivity, specificity, and positive likelihood ratio and negative likelihood ratio for predict the outcome of patients with sepsis at optimal cut-off points of MAP, ALB, and APACHE II are listed in Table 4. The optimal cut-off point of prediction probability of combined indicators was 0.381 . At this time, the false positive rate was 0.195 . If the probability obtained according to the logistic regression equation $\left(P=1 /\left[1+e^{-(5.312-0.077 \times 1+}\right.\right.$ $0.200 \times 2-0.125 \times 3)]$ ) was greater than 0.381 , it indicated the patient with sepsis had a high risk of death and should be paid great attention.

Table 4

Performance of each biomarker for evaluating the prognosis

\begin{tabular}{|c|c|c|c|c|c|c|c|}
\hline & $\beta$ & $\begin{array}{l}\text { Optimal cut-off } \\
\text { value }\end{array}$ & Sensitivity & Specificity & PLR & NLR & $\begin{array}{l}\text { Youden } \\
\text { index }\end{array}$ \\
\hline MAP & -0.077 & 73.0 & 0.659 & 0.756 & 3.098 & 0.323 & 0.415 \\
\hline APACHE II & 0.200 & 19.5 & 0.707 & 0.902 & 9.204 & 0.109 & 0.610 \\
\hline ALB & -0.125 & 32.5 & 0.927 & 0.329 & 0.490 & 2.039 & 0.256 \\
\hline $\begin{array}{l}\text { MAP + ALB + } \\
\text { APACHE ॥ }\end{array}$ & - & $0.381^{a}$ & 0.829 & 0.805 & 4.128 & 0.242 & 0.634 \\
\hline \multicolumn{8}{|c|}{$\begin{array}{l}\text { Abbreviations: MAP, mean arterial pressure; APACHE II, acute physiology and chronic health } \\
\text { evaluation; ALB, albumin. Logistic regression equation: } P=1 /\left[1+\mathrm{e}^{-(5.312-0.077 X 1+0.200 \times 2-0.125 \times 3)}\right] \\
\text { (X1:MAP, X2:APACHE II,X3:ALB). }\end{array}$} \\
\hline \multicolumn{8}{|c|}{ a:The probability greater than 0.381 indicated the patient with sepsis had a high risk of death. } \\
\hline
\end{tabular}

\section{Discussion}

At present, the main controversial topics regarding the early management of sepsis are the lack of a robust definition and reliable markers that can assist in the early identification of sepsis and the development of a therapeutic strategy. In this retrospective analysis, we found that the arterial blood lactate level, MAP, albumin level, SOFA score, and APACHE II score were risk factors for mortality in the univariate analysis, of which the MAP, albumin level, and APACHE II score had high prognostic value. Therefore, the above clinical parameters were analyzed in detail.

Blood lactate levels indicate organ dysfunction. Septic patients have decreased tissue perfusion and cellular hypoxia, in this context, the energy supply depends on anaerobic glycolysis, and the production of lactate gradually increases. Normally, the liver can further convert this lactic acids, but impaired liver function reduces lactate clearance.[11, 12] In critically ill patients, lactate can be used as an indicator of the cumulative oxygen load and as a prognostic indicator. Mikkelsen et al reported that the initial lactate 
level was independently associated with mortality in patients with severe sepsis (OR $1.34,95 \% \mathrm{Cl} 1.30-$ $1.40, P=0.001)$. [13] Our study showed that the lactate level was a risk factor for mortality in patients with sepsis (OR 1.482, 95\% Cl 1.105-1.988), but it was not an independent factor. The difference between the two studies may be due to the larger sample size and fewer confounders in the former study. To date, the efficacy of lactate for the guidance of resuscitation has been reported because of its prognostic value,[14] but multicenter randomized trials have shown that fluid resuscitation is not beneficial in septic shock patients with or without hyperlactatemia.[15, 16] In addition, when hyperlactatemia is not caused by hypoperfusion, fluid resuscitation and lactate removal may be harmful. Therefore, high lactate levels should be interpreted on a patient-by-patient basis.

The main physiological functions of serum albumin are the regulation of the plasma colloid osmotic pressure and capillary membrane permeability, as well as the binding and transport of ligands. As a reservoir and carrier of many endogenous and exogenous compounds with antioxidant and circulatory protective properties, albumin is involved in free radical scavenging.[17] Current studies have shown that protein metabolism can indicate not only liver function but also prognosis in patients with critical illness. In a comprehensive meta-analysis of 90 cohort studies including 291,433 patients with critical illness, the efficacy of hypoalbuminemia as a predictor of the outcome was evaluated by multivariate analysis, which showed that a $10 \mathrm{~g} / \mathrm{L}$ decrease in the serum albumin concentration was associated with a significant $137 \%$ increase in mortality.[18] Similarly, our study showed that the serum albumin level was an independent risk factor for sepsis-related mortality (adjusted OR $0.755,95 \% \mathrm{Cl} 0.583-0.979$ ), and the AUC was 0.641 . All of the above findings indicate that albumin, as a nutritional indicator, can predict the later outcome of the disease. To ameliorate hypoalbuminemia, several albumin replacement studies have been conducted, such as the SAFE, CRISTAL, and ALBIOS projects, but these interventions have not reduced mortality of septic patients. One thing can be sure is that nutritional therapy is recommended in patients with sepsis as early as possible, as it may help improve hypoalbuminemia, maintain normal vascular permeability, and reduce mortality to some extent.[19-21]

The international guidelines define severe sepsis as a subset of sepsis cases wherein, despite adequate fluid resuscitation, patients have hypotension requiring vasopressors to maintain an MAP above 65 $\mathrm{mmHg}$. This hemodynamic indicator is a simple, convenient, and more accurate reflection of the perfusion level. Decreased tissue perfusion worsens clinical outcomes by affecting organ function.[5] In a similarly designed study involving 274 patients with sepsis and septic shock, the results were adjusted for disease severity. Patients with one or more hypotensive episodes during which the MAP was below 60 $\mathrm{mmHg}$ for at least $2 \mathrm{~min}$ had higher mortality at day 28 than those without hypotension. [22] However, the SEPSISPAM trial, a large prospective randomized controlled study that compared septic shock patients with high and low MAP, showed no difference in mortality between the two groups.[23] Our study showed that MAP was an independent risk factor for mortality in patients with sepsis (adjusted OR $0.889,95 \% \mathrm{Cl}$ $0.822-0.961)$, and the area under the ROC curve was $0.747,(95 \% \mathrm{Cl} 0.654-0.839)$, indicating that it could be used as a prognostic indicator and resuscitation goal for patients with sepsis. It has been shown that sepsis is associated with altered microcirculation.[24,25] The relationship between systemic hemodynamics and microcirculatory blood flow is complex. An increase in MAP levels above $65 \mathrm{mmHg}$ 
may not alter microvascular perfusion per se, and conditions in which MAP has been corrected while microcirculatory abnormalities still persist often occur. However, it is important to note that the timely initiation of interventions may play a major role. DeBacker et al reported that microcirculation could be improved by fluid resuscitation in the early stage of sepsis.[25] Fluid resuscitation is the preferred method of MAP enhancement, and the SSC advocates for the use of crystalloids. In fact, fluid overload can lead to the worsening of myocardial contractility, acute pulmonary edema, and possibly death. Hence, patients with septic shock and left-sided ventricular-arterial uncoupling may require more rigorous fluid management. In addition, vasoactive agents are required when fluid resuscitation is ineffective in patients with septic shock. Norepinephrine is recommended by the SSC as the most commonly used vasopressor in patients with sepsis.[26] However, to date, no precise MAP target has been identified for septic shock, and more research is needed.[27] In conclusion, MAP affects the prognosis of patients, and the targeted treatment goal needs to be individualized. More attention should be given to the appropriate use of vasoactive drugs for sepsis.

Multiple organ dysfunction is associated with a high risk of mortality. The SOFA and APACHE II scores are two comparatively comprehensive scores used to assess organ function, both of which correlate well with the outcome of sepsis.

The APACHE II score is widely used for the evaluation of the systemic functioning in critically ill patients to predict the severity of the disease and to facilitate risk stratification. In 1985, the University of Washington Medical Center proposed the APACHE II scoring system in a study of 5030 cases in 13 ICUs across the United States performed by Knaus et al.[10] This score provides an initial picture of the patient's general condition in the ICU and helps predict the clinical outcome. One study showed that APACHE II score could be used to identify disease severity, and there were significant differences in mortality among patient groups stratified by the APACHE II score. Regression analysis showed that an APACHE II score $\geq 15$ and soluble urokinase-type plasminogen activator receptor (suPAR) $\geq 10.82 \mathrm{ng} / \mathrm{mL}$ were independently associated with adverse outcomes.[28] Our study also showed that this score was an independent risk factor for mortality in patients with sepsis, (adjusted OR 1.356, 95\% Cl 1.119-1.643), and it possessed some prognostic value, with an AUC of 0.839. Thus, the APACHE II score should be calculated daily for patients with sepsis in the ICU to accurately monitor the overall condition of these patients in a timely manner. This study also indicated that the predictive value of the combination of the APACHE II score, MAP, and albumin level was significantly better than those of the individual indicators, with an AUC of 0.890 . We recommend the use of these indicators in clinical practice.

The SOFA score can be used as one of the key indicator for the diagnosis of sepsis and is also included by the European Medicines Agency (EMA) as a treatment efficacy indicator and clinical endpoint in phase II clinical trials on new treatment options.[5] It can reflect the severity of the disease to a certain extent and has some suggestive value with regard to prognosis. In the past, the improvement in 28-day mortality has been used as a primary endpoint in sepsis trials, which may result in the underestimation of some of the potential beneficial effects of the initial intervention.[29] Pocock et al[30] proposed that the SOFA score could be selected as a surrogate endpoint when the primary outcome of the study failed. The 
usefulness of serial evaluation of the SOFA score for the prediction of the outcome in critically ill patients has been previously reported many times. However, those studies only provided a fixed SOFA score, which is not able to comprehensively reflect the condition of patients with sepsis. Then, DeGrooth et al put forward the idea of using the change in the SOFA score (i.e., the score at a fixed date after randomization minus the baseline score, or the maximum score during the ICU stay minus the baseline score) as an endpoint in studies evaluating the treatment effect in critically ill patients. Based on the 87 randomized controlled studies included in the analysis, the change in the SOFA score appeared to be reliably and consistently associated with mortality.[31] Additionally, a series of observational studies have shown a significant effect on mortality of a small changes in the SOFA scores.[32] Studies have shown that changes in the SOFA scores within 48 hours in patients with sepsis are associated with DIC, sudden cardiac arrest, and other serious illnesses in the ICU and emergency room.[33, 34] In our study, univariate regression results showed that the SOFA score was a risk factor for sepsis-related mortality, although the relationship was nonsignificant after accounting for confounders, probably because we used a fixed SOFA score. Therefore, the change in the SOFA score is suggested is suggested as a useful predictor, in agreement with the above studies.

There are some limitations in this study. First, it was a retrospective study including 123 patients. Prospective randomized trials with larger sample sizes are needed to more accurately analyze the factors predictive of mortality in patients with sepsis. Second, the influential factors included in this study are not exhaustive. Finally, some patients were in critical condition, died within 24 hours or were discharged automatically, leading to insufficient clinical data and possibly affecting the final results.

\section{Conclusion}

Sepsis is associated with a high mortality rate and seriously affects the long-term patient quality of life, which is an important consideration. In this study, we found that the combination of the MAP, albumin level, and APACHE II score was the best predictor of the risk of mortality, and we proposed regression formula calculation to more precisely reflect the risk of death.

\section{Abbreviations}

RICU: Respiratory intensive care unit; EICU: Emergency ICU; SICU: Surgical ICU; IL-27: interleukin-27; IL-6: interleukin-6; ROC: Receiver operating characteristic curve; AUC: Area under the curve; Cl: Confidence interval; ARDS: Acute respiratory distress syndrome; AKI: Acute kidney injury; DIC: Disseminated intravascular coagulation; APACHE II: Acute physiology and chronic health evaluation II; MAP: Mean arterial pressure; SSC: surviving Sepsis Campaign; WBC: White blood cell; PT: Prothrombin time; TT: Thrombin time; INR: International normalized ratio; APTT: Activated partial thromboplastin time; CRP: Creactive protein; GCS: Glasgow Coma Scale; D-D: D-dimer; SOFA: Sequential organ failure assessment; APS: Acute Physiology Score; CHS: Chronic Health Evaluation; EMA: European Medicines Agency; CKD: Chronic kidney disease; suPAR: Soluble urokinase-type plasminogen activator receptor; COPD: Chronic obstructive pulmonary disease; NLR: Neutrophil-to-lymphocyte ratio; AMI: Acute myocardial injury. 


\section{Declarations}

\section{Ethics approval and consent to participate}

This study was reviewed and approved by the Ethics Committee of Beijing Chao-Yang Hospital (2016-Ke115), and all methods were performed in accordance with the relevant guidelines. Waiver of consent was granted by the Institutional Review Board since this was a retrospective study.

\section{Consent for publication}

Not applicable.

\section{Availability of data and materials}

All the data and materials are available from the corresponding author by request.

\section{Competing interests}

The authors declare that they have no competing interests.

\section{Funding}

This research did not receive any specific grant from funding agencies in the public, commercial, or notfor-profit sectors.

\section{Authors' contributions}

Huifeng Wang: Methodology, Verification, Original draft. Zhiling Zhao: Formal analysis, Supervision, Data curation. Zhao-hui Tong: Conceptualization, Supervision, Project administration. All authors read and approved the final manuscript.

\section{Acknowledgements}

We would like to thank all patients with sepsis and relevant doctor.

\section{References}

1. Gaieski DF, Edwards JM, Kallan MJ, Carr BG: Benchmarking the incidence and mortality of severe sepsis in the United States. Critical care medicine 2013, 41(5):1167-1174.

2. Rhodes A, Evans LE, Alhazzani W, Levy MM, Antonelli M, Ferrer R, Kumar A, Sevransky JE, Sprung CL, Nunnally ME et al: Surviving Sepsis Campaign: International Guidelines for Management of Sepsis and Septic Shock: 2016. Intensive care medicine 2017, 43(3):304-377.

3. Liu V, Escobar GJ, Greene JD, Soule J, Whippy A, Angus DC, Iwashyna TJ: Hospital deaths in patients with sepsis from 2 independent cohorts. Jama 2014, 312(1):90-92. 
4. Perman SM, Goyal M, Gaieski DF: Initial emergency department diagnosis and management of adult patients with severe sepsis and septic shock. Scandinavian journal of trauma, resuscitation and emergency medicine 2012, 20:41.

5. Singer M, Deutschman CS, Seymour CW, Shankar-Hari M, Annane D, Bauer M, Bellomo R, Bernard GR, Chiche JD, Coopersmith CM et al: The Third International Consensus Definitions for Sepsis and Septic Shock (Sepsis-3). Jama 2016, 315(8):801-810.

6. Fleischmann C, Scherag A, Adhikari NK, Hartog CS, Tsaganos T, Schlattmann P, Angus DC, Reinhart K: Assessment of Global Incidence and Mortality of Hospital-treated Sepsis. Current Estimates and Limitations. American journal of respiratory and critical care medicine 2016, 193(3):259-272.

7. Jeon K, Na SJ, Oh DK, Park S, Choi EY, Kim SC, Seong GM, Heo J, Chang Y, Kwack WG et al: Characteristics, management and clinical outcomes of patients with sepsis: a multicenter cohort study in Korea. Acute and critical care 2019, 34(3):179-191.

8. Kim MH, Choi JH: An Update on Sepsis Biomarkers. Infection \& chemotherapy 2020, 52(1):1-18.

9. Cecconi M, Evans L, Levy M, Rhodes A: Sepsis and septic shock. Lancet (London, England) 2018, 392(10141):75-87.

10. Knaus WA, Draper EA, Wagner DP, Zimmerman JE: APACHE II: a severity of disease classification system. Critical care medicine 1985, 13(10):818-829.

11. American College of Chest Physicians/Society of Critical Care Medicine Consensus Conference: definitions for sepsis and organ failure and guidelines for the use of innovative therapies in sepsis. Critical care medicine 1992, 20(6):864-874.

12. Levy MM, Fink MP, Marshall JC, Abraham E, Angus D, Cook D, Cohen J, Opal SM, Vincent JL, Ramsay G: 2001 SCCM/ESICM/ACCP/ATS/SIS International Sepsis Definitions Conference. Intensive care medicine 2003, 29(4):530-538.

13. Houwink AP, Rijkenberg S, Bosman RJ, van der Voort PH: The association between lactate, mean arterial pressure, central venous oxygen saturation and peripheral temperature and mortality in severe sepsis: a retrospective cohort analysis. Critical care (London, England) 2016, 20:56.

14. Jones AE, Shapiro NI, Trzeciak S, Arnold RC, Claremont HA, Kline JA: Lactate clearance vs central venous oxygen saturation as goals of early sepsis therapy: a randomized clinical trial. Jama 2010, 303(8):739-746.

15. Yealy DM, Kellum JA, Huang DT, Barnato AE, Weissfeld LA, Pike F, Terndrup T, Wang HE, Hou PC, LoVecchio F et al: A randomized trial of protocol-based care for early septic shock. The New England journal of medicine 2014, 370(18):1683-1693.

16. Peake SL, Delaney A, Bailey M, Bellomo R, Cameron PA, Cooper DJ, Higgins AM, Holdgate A, Howe $\mathrm{BD}$, Webb SA et al: Goal-directed resuscitation for patients with early septic shock. The New England journal of medicine 2014, 371(16):1496-1506.

17. Fanali G, di Masi A, Trezza V, Marino M, Fasano M, Ascenzi P: Human serum albumin: from bench to bedside. Molecular aspects of medicine 2012, 33(3):209-290. 
18. Vincent JL, Dubois MJ, Navickis RJ, Wilkes MM: Hypoalbuminemia in acute illness: is there a rationale for intervention? A meta-analysis of cohort studies and controlled trials. Annals of surgery 2003, 237(3):319-334.

19. Finfer S, Bellomo R, Boyce N, French J, Myburgh J, Norton R: A comparison of albumin and saline for fluid resuscitation in the intensive care unit. The New England journal of medicine 2004, 350(22):2247-2256.

20. Evans S: Effects of fluid resuscitation with colloids vs. crystalloids on mortality in critically ill patients presenting with hypovolemic shock. Journal of the Intensive Care Society 2015, 16(2):169171.

21. Caironi P, Tognoni G, Masson S, Fumagalli R, Pesenti A, Romero M, Fanizza C, Caspani L, Faenza S, Grasselli $\mathrm{G}$ et al: Albumin replacement in patients with severe sepsis or septic shock. The New England journal of medicine 2014, 370(15):1412-1421.

22. Dünser MW, Takala J, Ulmer H, Mayr VD, Luckner G, Jochberger S, Daudel F, Lepper P, Hasibeder WR, Jakob SM: Arterial blood pressure during early sepsis and outcome. Intensive care medicine 2009, 35(7):1225-1233.

23. Asfar P, Meziani F, Hamel JF, Grelon F, Megarbane B, Anguel N, Mira JP, Dequin PF, Gergaud S, Weiss $\mathrm{N}$ et al: High versus low blood-pressure target in patients with septic shock. The New England journal of medicine 2014, 370(17):1583-1593.

24. De Backer D, Donadello K, Taccone FS, Ospina-Tascon G, Salgado D, Vincent JL: Microcirculatory alterations: potential mechanisms and implications for therapy. Annals of intensive care 2011, 1(1):27.

25. De Backer D, Creteur J, Preiser JC, Dubois MJ, Vincent JL: Microvascular blood flow is altered in patients with sepsis. American journal of respiratory and critical care medicine 2002, 166(1):98-104.

26. Hamzaoui O, Georger JF, Monnet X, Ksouri H, Maizel J, Richard C, Teboul JL: Early administration of norepinephrine increases cardiac preload and cardiac output in septic patients with life-threatening hypotension. Critical care (London, England) 2010, 14(4):R142.

27. Waechter J, Kumar A, Lapinsky SE, Marshall J, Dodek P, Arabi Y, Parrillo JE, Dellinger RP, Garland A: Interaction between fluids and vasoactive agents on mortality in septic shock: a multicenter, observational study. Critical care medicine 2014, 42(10):2158-2168.

28. Liu X, Shen Y, Li Z, Fei A, Wang H, Ge Q, Pan S: Prognostic significance of APACHE II score and plasma suPAR in Chinese patients with sepsis: a prospective observational study. $B M C$ anesthesiology 2016, 16(1):46.

29. Iba T, Arakawa M, Ohchi Y, Arai T, Sato K, Wada H, Levy JH: Prediction of Early Death in Patients With Sepsis-Associated Coagulation Disorder Treated With Antithrombin Supplementation. Clinical and applied thrombosis/hemostasis: official journal of the International Academy of Clinical and Applied Thrombosis/Hemostasis 2018, 24(9_suppl):145s-149s.

30. Pocock SJ, Stone GW: The Primary Outcome Fails - What Next? The New England journal of medicine 2016, 375(9):861-870. 
31. Ferreira FL, Bota DP, Bross A, Mélot C, Vincent JL: Serial evaluation of the SOFA score to predict outcome in critically ill patients. Jama 2001, 286(14):1754-1758.

32. García-Gigorro R, Sáez-de la Fuente I, Marín Mateos H, Andrés-Esteban EM, Sanchez-Izquierdo JA, Montejo-González JC: Utility of SOFA and $\Delta$-SOFA scores for predicting outcome in critically ill patients from the emergency department. European journal of emergency medicine: official journal of the European Society for Emergency Medicine 2018, 25(6):387-393.

33. Iba T, Arakawa M, Mochizuki K, Nishida O, Wada H, Levy JH: Usefulness of Measuring Changes in SOFA Score for the Prediction of 28-Day Mortality in Patients With Sepsis-Associated Disseminated Intravascular Coagulation. Clinical and applied thrombosis/hemostasis: official journal of the International Academy of Clinical and Applied Thrombosis/Hemostasis 2019, 25:1076029618824044.

34. Matsuda J, Kato S, Yano H, Nitta G, Kono T, Ikenouchi T, Murata K, Kanoh M, Inamura Y, Takamiya T et al: The Sequential Organ Failure Assessment (SOFA) score predicts mortality and neurological outcome in patients with post-cardiac arrest syndrome. Journal of cardiology 2020, 76(3):295-302.

\section{Figures}

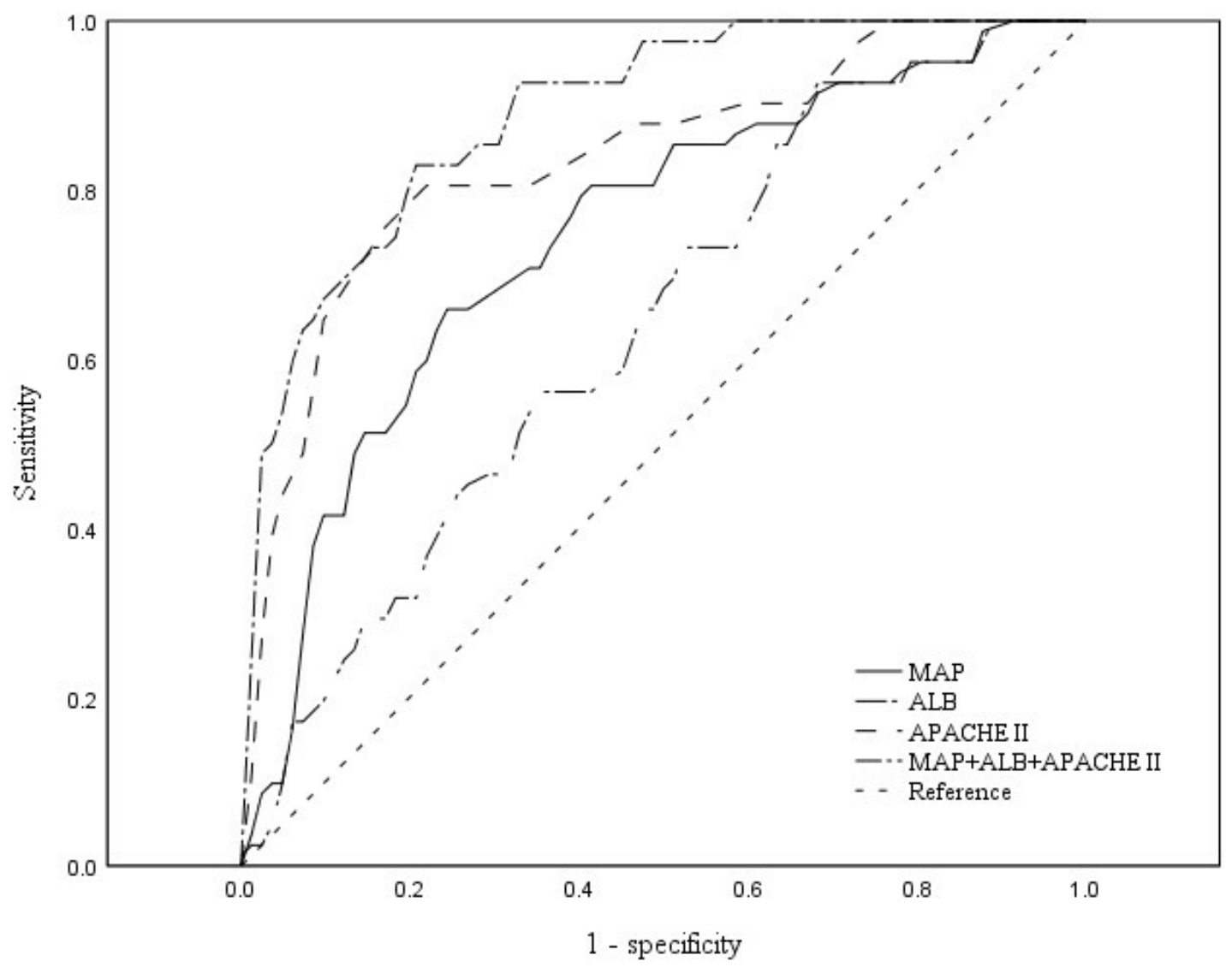

Figure 1 
The ROC of MAP, ALB, and APACHE II for ability to evaluate the prognose. The AUC is $0.747(95 \% \mathrm{Cl}$ 0.654-0.839) for MAP, 0.641 (95\% Cl 0.541-0.741) for ALB, 0.839 (95\% Cl 0.760-0.917) for APACHE II, and 0.890 (95\% $\mathrm{Cl} 0.832-0.948)$ for the combination of them. The AUC value of APACHE II is significantly higher than of both MAP $(p<0.001)$ and ALB $(p=0.011)$. The AUC value of the combination of them is the highest. Abbreviations: MAP, mean arterial pressure; APACHE II, acute physiology and chronic health evaluation; ALB, albumin; ROC, receiver operating characteristic curve; $A U C$, area under the curve; $\mathrm{Cl}$, confidence interval. 